Ambiente \& Água - An Interdisciplinary Journal of Applied Science
ISSN 1980-993X - doi:10.4136/1980-993X
www.ambi-agua.net
E-mail: ambi-agua@agro.unitau.br

\title{
Variabilidade espaço-temporal da temperatura e difusividade térmica do solo de Lagoa Seca-PB
}

\author{
doi: 10.4136/ambi-agua.1474 \\ Received: 30 Jul. 2014; Accepted: 19 Sep. 2014

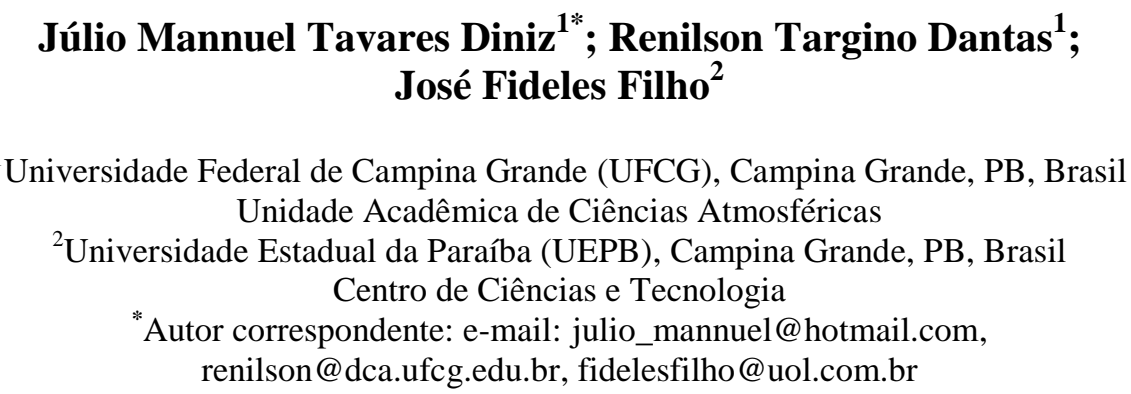

\section{RESUMO}

O objetivo deste trabalho foi investigar a variabilidade espaço-temporal da temperatura e difusividade térmica do solo de Lagoa Seca-PB. Para alcançar este objetivo foram utilizados sensores de temperatura, programados a operar ininterruptamente ao longo dos ciclos diários, instalados em quatro profundidades distintas do solo. Estes, por sua vez, monitoraram o comportamento térmico do solo durante todo o ano de 2010 e 2011. A fim de estimar a difusividade térmica do solo foram empregados três métodos distintos: método da amplitude, método do arco tangente e método logarítmico. O regime térmico do solo, no que diz respeito às horas e aos meses de ocorrência das máximas e mínimas temperaturas, apresentaram-se bastante semelhantes em ambos os anos. $\mathrm{O}$ método do arco tangente, para o caso específico da camada compreendida entre 5 e $50 \mathrm{~cm}$ de profundidade, não é adequado para a estimativa da difusividade térmica do solo.

Palavras-chave: difusividade térmica, física do solo, regime térmico, temperatura do solo.

\section{Spatial-temporal variability of temperature and soil thermal diffusivity from Lagoa Seca-PB}

\begin{abstract}
This work investigated the spatial-temporal variability of temperature and thermal diffusivity of Lagoa Seca-PB soil. To achieve this, we used temperature sensors, programmed to operate continuously over the daily cycles, installed in four distinct depths of soil. We monitored the soil's thermal behavior throughout a one-year period from 2010 to 2011. In order to estimate the soil's thermal diffusivity, we employed three distinct methods: the amplitude method, the arctangent method and the logarithmic method. The soil's thermal regime, i.e., the hours and months of occurrence of maximum and minimum temperatures, was very similar in both years. The arctangent method was inadequate to estimate the soil's thermal diffusivity, considering the specific layer between 5 and $50 \mathrm{~cm}$ depth.
\end{abstract}

Keywords: soil physics, soil temperature, thermal diffusivity, thermal regime. 


\section{INTRODUÇÃO}

De acordo com Lal e Shukla (2004), um dos elementos de maior importância para o desenvolvimento das plantas é a temperatura do solo. A atividade microbiológica poderá ser interrompida, as sementes poderão não germinar e as plantas não se desenvolverem, caso o solo não apresente uma temperatura dentro dos limites fisiológicos dos processos envolvidos (Prevedello, 1996). Esta também intervém diretamente nas propriedades e movimentação da água no interior do solo, na difusão dos solutos e dos gases, nas reações químicas e em uma série de outros processos importantes (Reichardt e Timm, 2004).

A temperatura do solo está em constante mudança visto que este é continuamente perturbado pela "entrada" de calor mediante incidência de radiação solar como também pela "saída" através de processos físicos como a evaporação (Diniz et al., 2014). A variação da temperatura do solo que uma planta consegue suportar é relativamente ampla, no entanto, seu desenvolvimento é seriamente comprometido a partir do momento em que o solo passa a assumir temperaturas abaixo ou acima de certos valores extremos (Hillel, 2004).

Quando uma mesma quantidade de energia está disponível para solos distintos o processo de aquecimento e resfriamento pode ser bastante variável em virtude de suas propriedades térmicas específicas (Diniz et al., 2013b). Basicamente, as propriedades térmicas dos solos que intervêm diretamente em seu regime de temperatura são denominadas de calor específico, condutividade térmica e difusividade térmica. A difusividade térmica do solo está diretamente relacionada à capacidade desse meio em conduzir calor através de seu perfil vertical, ou seja, é um indicativo da rapidez com que o calor difunde-se no solo. Esta propriedade é função da constituição, granulometria, densidade e estrutura do solo (Silans et al., 2006).

Sabendo-se da sua importância e uma vez que cada tipo de solo possui características específicas, faz-se necessário que sejam identificadas para cada situação particular as suas propriedades térmicas (Danelichen e Biudes, 2011). Até então, poucos estudos dessa natureza foram realizados pela comunidade científica para os solos do Brasil (Diniz et al., 2013c). Portanto, a fim de contribuir para o desenvolvimento desta área científica, o presente trabalho tem como objetivo investigar a variabilidade espaço-temporal das temperaturas e difusividade térmica do solo de Lagoa Seca-PB.

\section{MATERIAL E MÉTODOS}

\subsection{Descrição da área de estudo}

$\mathrm{O}$ estudo foi desenvolvido na Estação Experimental de Lagoa Seca $\left(7,16^{\circ} \mathrm{S} ; 35,85^{\circ} \mathrm{O}\right.$ e altitude média de $634 \mathrm{~m}$ ), pertencente à Empresa Estadual de Pesquisa Agropecuária da Paraíba - EMEPA/PB. Esta se encontra situada no Sítio Imbaúba, mesorregião do agreste paraibano, cerca de $15 \mathrm{~km}$ da cidade de Campina Grande-PB. Possui uma área total de 110 ha (hectares), sendo esta composta por áreas experimentais e reservas florestais. Segundo a classificação climática de Köppen, o clima da região é do tipo tropical chuvoso, com estação seca no verão. A precipitação média anual é da ordem de $940 \mathrm{~mm}$ (maior concentração de chuva ocorre no período compreendido entre março e agosto), com uma umidade relativa e temperatura média anual do ar de aproximadamente $65 \%$ e $21,6^{\circ} \mathrm{C}$, respectivamente (Araújo et al., 2010).

De acordo com Araújo (2008), o solo da região é classificado como Neossolo Regolítico (também denominado Regossolo). Neossolos são solos pouco desenvolvidos e geralmente pouco profundos, em via de formação devido à reduzida atuação dos processos pedogenéticos (consequiência da resistência do material de origem ao intemperismo, condição climática ou geomorfológica) ou ao pouco tempo de ação dos agentes intempéricos e pedológicos (Pedron, 2007). O Neossolo Regolítico é um tipo de solo que apresenta textura arenosa e baixa capacidade de adsorção de nutrientes, quando comparado com solos argilosos 
(Tito et al., 1997); naturalmente, possui baixo teor de matéria orgânica e nitrogênio que diminuem, após alguns anos de uso.

\subsection{Dados}

Para o desenvolvimento da presente pesquisa foram utilizados dados obtidos ao longo dos anos de 2010 e 2011, a partir de uma estação meteorológica automática em funcionamento na Estação Experimental de Lagoa Seca. Com o objetivo de monitorar o regime térmico do solo foram utilizados sensores de temperatura operando ininterruptamente ao longo dos ciclos diários (o datalogger encontra-se programado a fornecer a magnitude média horária desta variável), instalados em quatro profundidades distintas do solo: 5, 15, 30 e 50 centímetros. Também se fez uso de um pluviômetro, instalado de acordo com as especificações da Organização Mundial de Meteorologia (OMM), a fim de obter informações acerca dos totais pluviométricos.

\subsection{Difusividade térmica do solo}

A fim de estimar a difusividade térmica considerou-se o solo um meio homogêneo, isotrópico, sem fontes ou sumidouros de calor, restringindo a análise apenas aos processos que ocorrem na direção vertical (Fideles Filho, 1988; Diniz et al., 2013a). A partir dessas considerações irá ser aplicado o tratamento clássico de condução de calor a um meio homogêneo proposto por Fourier que permite conhecer as amplitudes e fases da onda de calor.

Uma vez que a difusividade térmica é calculada para certas camadas específicas do solo, faz-se necessário que sejam destacados os critérios adotados nesta pesquisa. Devido à disponibilidade de dados as estimativas foram realizadas para as porções do solo denominadas de camada 1 (estende-se desde 5 até 15 centímetros de profundidade), camada 2 (estende-se desde 15 até 30 centímetros de profundidade), camada 3 (estende-se desde 30 até 50 centímetros de profundidade) e camada 4 (estende-se desde 5 até 50 centímetros de profundidade).

Mediante as informações e considerações anteriores foram empregados determinados métodos a fim de se estimar a difusividade térmica do solo, cujos detalhes podem ser encontrados em Gao et al. (2009). Os utilizados na presente pesquisa são denominados de método da amplitude, método do arco tangente e método logarítmico.

\subsection{Simulação da temperatura do solo}

A representação matemática mais simples do regime de temperatura do solo é obtida assumindo-se que, para todo o perfil vertical, a temperatura oscila como uma pura função harmônica (senoidal) do tempo em torno de um valor médio. Esta pode ser uma aproximação grosseira (as variações reais não são ordenadas), no entanto, em determinadas situações pode fornecer valores bastante satisfatórios. Partindo deste pressuposto, após algumas considerações e manipulações matemáticas, obtém-se a Equação 1.

$$
\mathrm{T}(\mathrm{z}, \mathrm{t})=\mathrm{T}_{\text {média }}+\mathrm{A}_{\mathrm{o}} \mathrm{e}^{(-\mathrm{z} / \mathrm{d})}\left[\sin \left(\omega \mathrm{t}-\frac{7 \pi}{12}-\frac{\mathrm{z}}{\mathrm{d}}\right)\right]
$$

em que:

$\mathrm{T}_{\text {média }}$ é a temperatura média da superfície,

$\mathrm{A}_{\mathrm{o}}$ é a amplitude da flutuação de temperatura da superfície,

$\mathrm{z}$ é a profundidade em análise,

t é o instante para o qual se deseja simular a temperatura, 
$\omega$ é a velocidade angular da Terra $\left(\omega=7,27 \times 10^{-5} \mathrm{~s}^{-1}\right) \mathrm{e}$

d é a profundidade de amortecimento.

A fim de empregar a Equação 1, uma vez que não há equipamento destinado a mensurar a temperatura da superfície, considera-se nesta pesquisa que a $\mathrm{T}_{\text {média }}$ é igual aquela obtida para a profundidade de $5 \mathrm{~cm}$. A profundidade de amortecimento é obtida a partir da Equação 2.

$$
d=\left(\frac{2 \alpha}{\omega}\right)^{1 / 2}
$$

em que:

$\omega$ é a velocidade angular da Terra e

$\alpha$ é a difusividade térmica do solo.

Verifica-se, portanto, que a única informação desconhecida e necessária para simular a temperatura do solo é aquela referente à difusividade térmica do presente meio.

A Equação 1, fazendo uso das magnitudes da difusividade térmica do solo obtidas mediante as considerações e métodos apresentados anteriormente, será utilizada na simulação da temperatura do solo para diferentes profundidades e cada hora do dia. Temperaturas próximas daquelas mensuradas indica que os métodos utilizados forneceram valores condizentes com a realidade (Diniz, 2014).

\subsection{Análise dos dados}

Após simular a temperatura do solo empregando as diferentes magnitudes da difusividade térmica, faz-se necessário verificar o quanto estes valores se aproximam daqueles obtidos pelos sensores térmicos. Diferentes indicadores estatísticos podem ser utilizados a fim de alcançar este objetivo. Os utilizados nesta pesquisa são aqueles denominados de coeficiente de correlação (r) e índice de concordância (d) cujos detalhes podem ser encontrados em Larson e Farber (2004) e Willmott et al. (1985), respectivamente.

$\mathrm{O}$ índice de confiança (c) também será utilizado na presente pesquisa a fim de verificar o desempenho da Equação 1, na simulação da temperatura do solo para diferentes profundidades e horas do dia. De acordo com Camargo e Sentelhas (1997), a magnitude deste pode ser obtida a partir da Equação 3.

$$
\mathrm{c}=\mathrm{rxd}
$$

em que:

r é o coeficiente de correlação e

d é o índice de concordância.

O desempenho da Equação 1, avaliado a partir do índice de confiança, será classificado de acordo com a Tabela 1 . 
Tabela 1. Classificação de desempenho com base no índice de confiança (Fonte: Zonta et al., 2012).

\begin{tabular}{cl}
\hline Valor de c & Desempenho \\
\hline$>0,85$ & Ótimo \\
0,76 a 0,85 & Muito bom \\
0,66 a 0,75 & Bom \\
0,61 a 0,65 & Mediano \\
0,51 a 0,60 & Sofrível \\
0,41 a 0,50 & Mau \\
$\leq 0,40$ & Péssimo \\
\hline
\end{tabular}

\section{RESULTADOS E DISCUSSÃO}

A partir dos dados de temperatura do solo tornou-se possível o estudo do regime térmico diário e anual do presente meio. Encontra-se exposto nas Figuras 1 e 2, para aquelas profundidades em estudo, o comportamento térmico do solo observado durante os anos de 2010 e 2011, respectivamente.

Verifica-se, a partir da análise das Figuras 1 e 2, que o comportamento térmico do solo observado em ambos os ciclos anuais são significativamente semelhantes. As temperaturas mais elevadas observadas em todo o perfil vertical foram aquelas oriundas dos primeiros e últimos meses dos respectivos anos (Janeiro-Março e Outubro-Dezembro). Para todo o período de análise as máximas foram registradas entre os dias 01 e 21 de março do ano de 2010, alcançando magnitudes da ordem dos $45^{\circ} \mathrm{C}$ (temperatura referente a profundidade de $5 \mathrm{~cm}$ ). Em contrapartida, as mínimas foram observadas em torno do mês de Junho, sendo registradas magnitudes da ordem dos $16^{\circ} \mathrm{C}$ (temperatura referente a profundidade de $5 \mathrm{~cm}$ ). Esse comportamento, uma vez que a responsável pelo aquecimento é a energia advinda do Sol, já era esperado visto que no verão (inverno) há uma maior (menor) disponibilidade de energia solar à superfície (Diniz et al., 2013d).

Uma vez que as regiões mais profundas foram as que apresentaram menor variabilidade térmica anual, pode-se considerar que estas grandezas estão inversamente relacionadas. Verifica-se, para a profundidade de $5 \mathrm{~cm}$, uma amplitude térmica em torno dos $29^{\circ} \mathrm{C}$ e $23^{\circ} \mathrm{C}$ para os ciclos anuais de 2010 e 2011, respectivamente. Em contrapartida, aquela referente à profundidade de $50 \mathrm{~cm}$, não chega a ultrapassar os $13^{\circ} \mathrm{C}$. $\mathrm{O}$ ciclo anual que apresentou maior variabilidade térmica foi aquele de 2010. A precipitação pluvial foi a principal responsável pelo fenômeno mencionado anteriormente, a registrada para todo o ano de 2010 foi quase que a metade daquela precipitada em 2011, uma vez que esta ocasiona uma alteração considerável no teor de umidade do solo e é capaz de impedir que uma parcela significativa da energia proveniente do Sol incida na superfície. De acordo com Carneiro et al. (2014), o regime pluviométrico exerce grande influência na incidência de radiação solar à superfície sendo, portanto, de grande contribuição para a variabilidade da temperatura do solo no decorrer do ano. Devido à disponibilidade energética pouco se alterar ao longo do ano em regiões próximas ao equador geográfico (baixas latitudes), a variabilidade anual das condições térmicas do solo não é acentuada como a oriunda de demais regiões (médias e altas latitudes). 

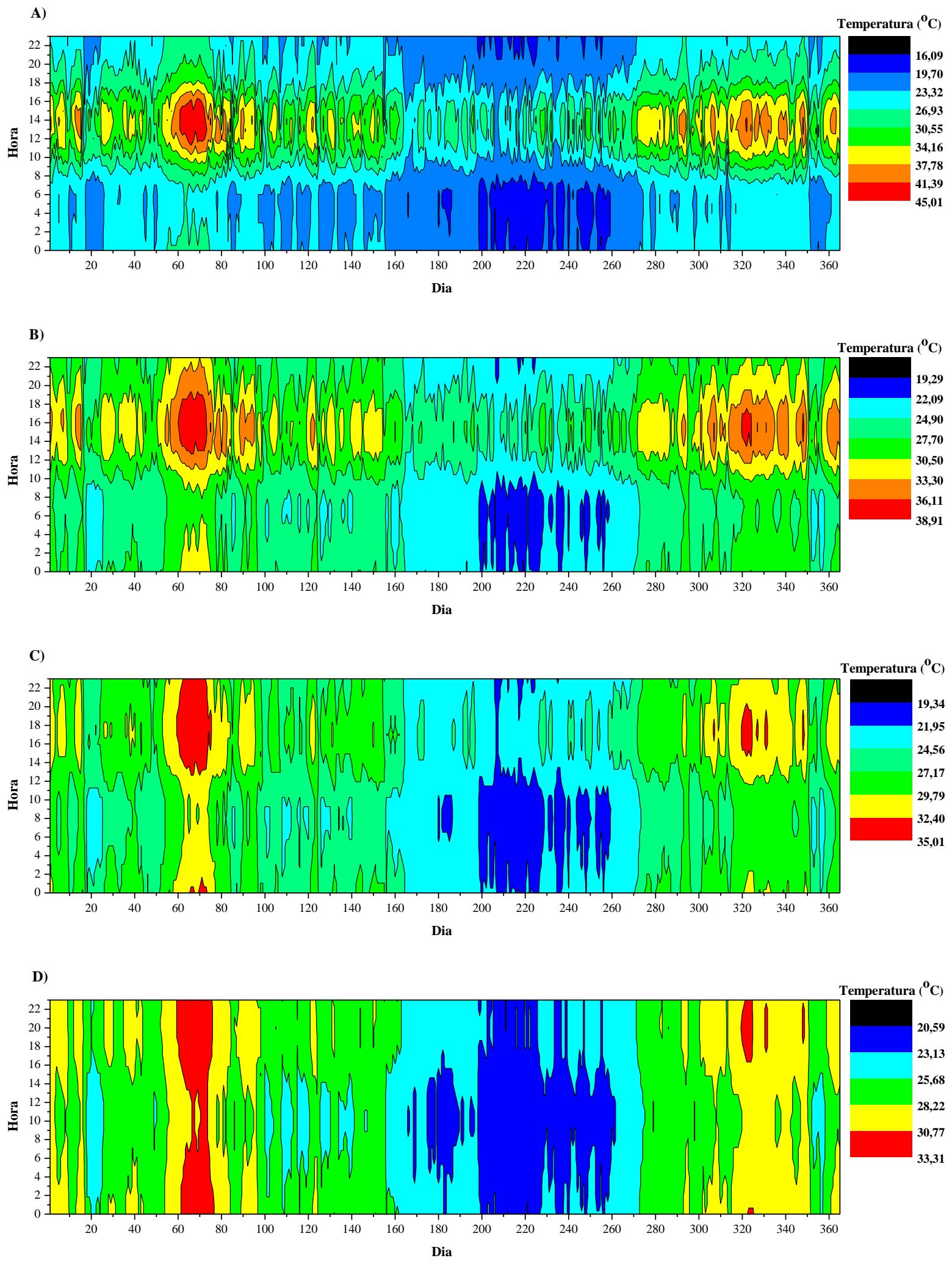

Figura 1. Comportamento térmico do solo de Lagoa Seca-PB, observado ao longo de todo o ano de 2010, referente às seguintes profundidades: $5 \mathrm{~cm}(\mathrm{~A}), 15 \mathrm{~cm}(\mathrm{~B}), 30 \mathrm{~cm}(\mathrm{C}) \mathrm{e} 50 \mathrm{~cm}(\mathrm{D})$. 

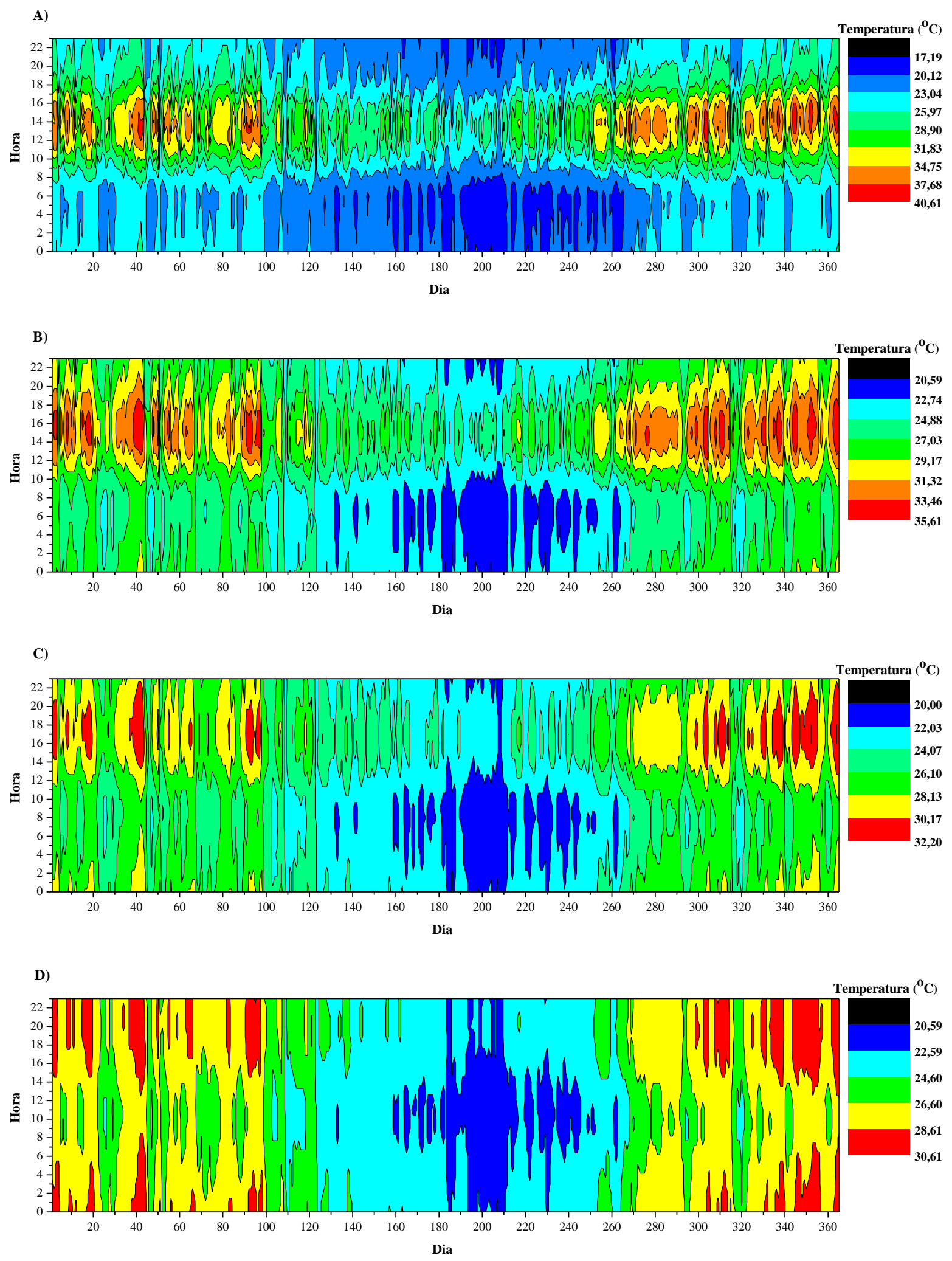

Figura 2. Comportamento térmico do solo de Lagoa Seca-PB, observado ao longo de todo o ano de 2011, referente às seguintes profundidades: $5 \mathrm{~cm}(\mathrm{~A}), 15 \mathrm{~cm}(\mathrm{~B}), 30 \mathrm{~cm}(\mathrm{C}) \mathrm{e} 50 \mathrm{~cm}(\mathrm{D})$.

A precipitação pluvial é capaz de alterar significativamente o comportamento térmico diário do solo. Na ausência deste fenômeno, verifica-se que os ciclos térmicos diários são bastante semelhantes, divergindo basicamente quanto às magnitudes das temperaturas. Neste caso específico, constata-se até meados das 10 horas da manhã o progressivo aumento das 
temperaturas à medida que se avança em profundidade, com os valores máximos sendo observados em $50 \mathrm{~cm}$. Após esse período, em conseqüência da incidência mais acentuada de radiação solar, a temperatura da superfície do solo e de regiões adjacentes passa a apresentar um significativo aumento de suas magnitudes. No entanto, à medida que se avança em profundidade verifica-se que a variabilidade térmica tende a diminuir (variações bastante sutis), de modo que as temperaturas permanecem quase que constante ao longo dos ciclos diários na profundidade de $50 \mathrm{~cm}$. Portanto, como já havia sido destacado anteriormente para o ciclo anual, ambas apresentam-se novamente inversamente relacionadas (quanto maior a profundidade, menor será a variabilidade térmica diária e anual). Segundo Azevedo e Galvani (2003), quanto mais próximo da superfície, maior é a amplitude térmica e mais imediata é a resposta da temperatura do solo à incidência da radiação solar. De acordo com Gasparim et al. (2005), pode-se definir a profundidade de $20 \mathrm{~cm}$ como a profundidade de transição entre as grandes e as pequenas variações da temperatura do solo. As regiões mais próximas a superfície apresentam maior amplitude térmica diária em decorrência da maior facilidade em ganhar (período diurno) e perder (período noturno) calor durante os ciclos diários.

As temperaturas mais elevadas observadas ao longo dos ciclos diários foram aquelas registradas em torno dos seguintes horários: 14 horas (profundidade de $5 \mathrm{~cm}$ ), 16 horas (profundidade de $15 \mathrm{~cm}$ ) e 18 horas (profundidade de $30 \mathrm{~cm}$ ). Em particular, para a profundidade de $50 \mathrm{~cm}$, estas foram registradas nas primeiras e últimas horas do ciclo diário. Oliveira et al. (2010), fazendo uso de dados obtidos em uma localidade relativamente próxima, verificaram que em regiões superficiais as máximas temperaturas são observadas entre as 13 e 14 horas. Ainda segundo estes autores, em profundidades intermediárias as máximas são observadas entre o fim da tarde e início da noite. Ao comparar à hora de ocorrência das máximas temperaturas do solo verifica-se, portanto, que estas não ocorrem simultaneamente ao longo de todo o perfil vertical. Este fenômeno está associado ao fato de que o solo exerce certa "resistência" a propagação de calor (quanto menor esta "resistência", menor será a defasagem de ocorrência das temperaturas máximas), variando de acordo com as suas propriedades térmicas específicas. Portanto, esta mudança em comparação a região mais superficial é consequência do fluxo de calor no interior do solo ocorrer de maneira relativamente lenta.

Os dados de temperatura foram utilizados para estimar a difusividade térmica diária média mensal do solo. Esta grandeza, mediante o emprego de métodos distintos, foi estimada para quatro camadas específicas do respectivo meio. Nas Tabelas 2 e 3 estão apresentados os valores obtidos a partir de dados coletados ao longo do ano de 2010 e 2011, respectivamente.

As estimativas da difusividade térmica do solo estão de acordo com aquelas obtidas em Rao et al. (2005), Oliveira et al. (2010), Danelichen e Biudes (2011), Diniz et al. (2013e). No que diz respeito à camada-1, com base na análise das Tabelas 2 e 3 , verifica-se que as mais elevadas magnitudes da difusividade térmica são aquelas oriundas do método do arco tangente. Neste caso específico, de acordo com o observado por Rao et al. (2005), apresentaram-se bastante semelhantes as estimativas provenientes dos métodos da amplitude e logarítmico. Para todo o ano de 2010, ainda com relação à camada-1, observa-se que as mais elevadas magnitudes são aquelas associadas ao mês de Junho. Portanto, comparados aqueles referentes aos demais meses deste ano, pode-se concluir que os ciclos diários pertencentes a este mês possuíram em média maior capacidade de conduzir calor através desta camada particular do solo. Este resultado é consequiência da elevada precipitação pluvial registrada neste mês (159,8 milímetros), o espaço poroso do solo passa então a ser preenchido por água, propiciando a esta maior capacidade de condução (vale ressaltar que a condutividade térmica da água é bastante superior a do ar). Uma vez que este fenômeno não foi observado nas camadas adjacentes, pode-se deduzir que a água não infiltrou de maneira considerável através do perfil vertical do solo. 
Tabela 2. Difusividade térmica diária média mensal do solo $\left(\times 10^{-6} \mathrm{~m}^{2} / \mathrm{s}\right)$, referente a quatro camadas específicas, estimada a partir de métodos distintos e de dados coletados ao longo do ano de 2010.

\begin{tabular}{|c|c|c|c|c|c|}
\hline \multirow{2}{*}{ Mês } & \multirow{2}{*}{ Método } & \multicolumn{4}{|c|}{ Camadas (m) } \\
\hline & & $0,05-0,15$ & $0,15-0,30$ & $0,30-0,50$ & $0,05-0,50$ \\
\hline \multirow[t]{3}{*}{ Janeiro } & Amplitude & 0,75 & 2,57 & 2,51 & 1,80 \\
\hline & Arco Tangente & 1,26 & 2,46 & 2,28 & 4,87 \\
\hline & Logarítmico & 0,70 & 2,21 & 2,86 & 1,77 \\
\hline \multirow[t]{3}{*}{ Fevereiro } & Amplitude & 0,83 & 2,72 & 3,56 & 2,16 \\
\hline & Arco Tangente & 1,33 & 2,66 & 2,60 & 4,25 \\
\hline & Logarítmico & 0,86 & 2,24 & 3,54 & 2,05 \\
\hline \multirow[t]{3}{*}{ Março } & Amplitude & 0,76 & 2,68 & 2,80 & 1,90 \\
\hline & Arco Tangente & 1,18 & 3,11 & 2,67 & 4,13 \\
\hline & Logarítmico & 0,83 & 2,43 & 3,34 & 2,03 \\
\hline \multirow[t]{3}{*}{ Abril } & Amplitude & 0,75 & 2,54 & 3,47 & 2,01 \\
\hline & Arco Tangente & 1,30 & 2,53 & 2,26 & 4,79 \\
\hline & Logarítmico & 0,70 & 2,46 & 3,70 & 1,99 \\
\hline \multirow[t]{3}{*}{ Maio } & Amplitude & 1,02 & 2,47 & 2,65 & 2,01 \\
\hline & Arco Tangente & 1,38 & 2,58 & 2,32 & 4,54 \\
\hline & Logarítmico & 1,08 & 2,17 & 2,89 & 2,03 \\
\hline \multirow[t]{3}{*}{ Junho } & Amplitude & 1,11 & 2,20 & 1,93 & 1,75 \\
\hline & Arco Tangente & 1,98 & 2,61 & 1,72 & 4,83 \\
\hline & Logarítmico & 1,09 & 1,96 & 2,07 & 1,73 \\
\hline \multirow[t]{3}{*}{ Julho } & Amplitude & 1,00 & 2,49 & 3,12 & 2,13 \\
\hline & Arco Tangente & 1,52 & 2,12 & 1,77 & 5,81 \\
\hline & Logarítmico & 0,92 & 2,57 & 2,47 & 1,92 \\
\hline \multirow[t]{3}{*}{ Agosto } & Amplitude & 1,09 & 2,59 & 3,19 & 2,24 \\
\hline & Arco Tangente & 1,61 & 2,31 & 2,18 & 4,68 \\
\hline & Logarítmico & 1,07 & 2,22 & 3,74 & 2,23 \\
\hline \multirow[t]{3}{*}{ Setembro } & Amplitude & 0,87 & 2,54 & 3,15 & 2,05 \\
\hline & Arco Tangente & 1,51 & 2,52 & 2,22 & 4,55 \\
\hline & Logarítmico & 0,88 & 2,27 & 2,78 & 1,90 \\
\hline \multirow[t]{3}{*}{ Outubro } & Amplitude & 0,59 & 2,05 & 2,88 & 1,62 \\
\hline & Arco Tangente & 1,28 & 2,33 & 2,20 & 5,10 \\
\hline & Logarítmico & 0,62 & 1,74 & 3,09 & 1,62 \\
\hline \multirow[t]{3}{*}{ Novembro } & Amplitude & 0,92 & 2,91 & 3,04 & 2,15 \\
\hline & Arco Tangente & 1,15 & 2,73 & 2,55 & 4,52 \\
\hline & Logarítmico & 0,91 & 2,65 & 3,28 & 2,15 \\
\hline \multirow[t]{3}{*}{ Dezembro } & Amplitude & 0,91 & 2,56 & 3,03 & 2,06 \\
\hline & Arco Tangente & 1,53 & 2,78 & 2,27 & 4,29 \\
\hline & Logarítmico & 1,01 & 2,28 & 3,17 & 2,09 \\
\hline
\end{tabular}


Tabela 3. Difusividade térmica diária média mensal do solo $\left(\times 10^{-6} \mathrm{~m}^{2} / \mathrm{s}\right)$, referente a quatro camadas específicas, estimada a partir de métodos distintos e de dados coletados ao longo do ano de 2011.

\begin{tabular}{|c|c|c|c|c|c|}
\hline \multirow{2}{*}{ Mês } & \multirow{2}{*}{ Método } & \multicolumn{4}{|c|}{ Camadas (m) } \\
\hline & & $0,05-0,15$ & 0,15-0,30 & $0, \mathbf{3 0 - 0 , 5 0}$ & $0,05-0,50$ \\
\hline \multirow[t]{3}{*}{ Janeiro } & Amplitude & 1,14 & 3,09 & 2,92 & 2,31 \\
\hline & Arco Tangente & 1,59 & 2,70 & 1,91 & 4,79 \\
\hline & Logarítmico & 1,17 & 2,88 & 2,99 & 2,30 \\
\hline \multirow[t]{3}{*}{ Fevereiro } & Amplitude & 1,06 & 3,00 & 3,55 & 2,41 \\
\hline & Arco Tangente & 1,66 & 2,54 & 1,97 & 4,74 \\
\hline & Logarítmico & 1,07 & 2,46 & 4,04 & 2,38 \\
\hline \multirow[t]{3}{*}{ Março } & Amplitude & 0,89 & 2,73 & 3,70 & 2,24 \\
\hline & Arco Tangente & 1,97 & 2,75 & 1,83 & 4,53 \\
\hline & Logarítmico & 1,05 & 2,46 & 4,00 & 2,35 \\
\hline \multirow[t]{3}{*}{ Abril } & Amplitude & 1,03 & 2,66 & 4,09 & 2,42 \\
\hline & Arco Tangente & 1,73 & 2,75 & 1,79 & 4,83 \\
\hline & Logarítmico & 1,17 & 2,40 & 4,93 & 2,59 \\
\hline \multirow[t]{3}{*}{ Maio } & Amplitude & 0,98 & 3,02 & 3,43 & 2,33 \\
\hline & Arco Tangente & 1,63 & 2,48 & 1,50 & 5,98 \\
\hline & Logarítmico & 1,13 & 2,38 & 3,87 & 2,36 \\
\hline \multirow[t]{3}{*}{ Junho } & Amplitude & 0,92 & 2,74 & 4,21 & 2,37 \\
\hline & Arco Tangente & 1,49 & 2,66 & 1,93 & 4,90 \\
\hline & Logarítmico & 0,85 & 2,70 & 4,48 & 2,34 \\
\hline \multirow[t]{3}{*}{ Julho } & Amplitude & 0,97 & 2,64 & 4,35 & 2,41 \\
\hline & Arco Tangente & 1,66 & 2,74 & 1,33 & 6,31 \\
\hline & Logarítmico & 0,94 & 2,45 & 3,14 & 2,08 \\
\hline \multirow[t]{3}{*}{ Agosto } & Amplitude & 1,06 & 2,49 & 2,87 & 2,10 \\
\hline & Arco Tangente & 1,89 & 3,02 & 1,34 & 5,67 \\
\hline & Logarítmico & 1,05 & 2,23 & 2,67 & 1,97 \\
\hline \multirow[t]{3}{*}{ Setembro } & Amplitude & 0,95 & 2,95 & 2,67 & 2,08 \\
\hline & Arco Tangente & 1,72 & 3,21 & 2,19 & 3,97 \\
\hline & Logarítmico & 0,96 & 2,60 & 2,65 & 2,00 \\
\hline \multirow[t]{3}{*}{ Outubro } & Amplitude & 0,94 & 3,19 & 2,79 & 2,15 \\
\hline & Arco Tangente & 1,71 & 3,40 & 2,33 & 3,76 \\
\hline & Logarítmico & 0,99 & 2,81 & 2,83 & 2,13 \\
\hline \multirow[t]{3}{*}{ Novembro } & Amplitude & 0,95 & 3,87 & 3,07 & 2,36 \\
\hline & Arco Tangente & 1,55 & 3,42 & 2,22 & 3,99 \\
\hline & Logarítmico & 0,89 & 3,54 & 3,15 & 2,28 \\
\hline \multirow[t]{3}{*}{ Dezembro } & Amplitude & 0,74 & 4,04 & 3,28 & 2,23 \\
\hline & Arco Tangente & 1,39 & 3,55 & 2,53 & 3,81 \\
\hline & Logarítmico & 0,76 & 3,83 & 3,35 & 2,24 \\
\hline
\end{tabular}


Com relação à camada-2, para ambos os anos de análise, verifica-se que as mínimas magnitudes da difusividade térmica são aquelas oriundas do método do logarítmico. Não se pode destacar entre os demais métodos, visto que não há uma expressiva predominância, aquele que fornece as máximas magnitudes para esta camada. As estimativas referentes a esta, obtidas a partir dos métodos da amplitude e arco tangente, mostraram-se bastante semelhantes em determinados meses de ambos os anos. Ao analisar as estimativas associadas à camada-3, para grande parte dos meses de ambos os anos, constata-se que as mais elevadas magnitudes são aquelas provenientes do método do logarítmico. Segundo os métodos da amplitude e logarítmico, ao comparar com as demais camadas, esta é a que apresenta maior capacidade de conduzir calor através de seu perfil vertical.

No caso específico da camada-4, de modo similar à camada-1, as magnitudes mais elevadas são aquelas oriundas do método do arco tangente. No entanto, estas magnitudes são superiores as obtidas para as demais camadas a partir do emprego deste método. Por uma questão de lógica, visto que neste caso está sendo considerado todo o perfil vertical do solo, esperava-se que estas estivessem entre aquelas oriundas das demais camadas. Este aspecto é observado ao examinar as estimativas provenientes dos demais métodos. Portanto, com base na análise dos resultados, pode-se constatar que não são satisfatórias as estimativas obtidas a partir do método do arco tangente para a camada-4. O fato de está sendo considerada uma camada de espessura apreciável, visto que nas demais este se adéqua a estimativa desta grandeza, deve ser a principal causa deste erro. Bellaver (2010), ao estimar a difusividade térmica para uma camada mais espessa que as demais (estendendo-se desde 0,01 até 0,30 metros de profundidade), verificou que o método do arco tangente passou a superestimar a magnitude desta grandeza para todos os ciclos diários em estudo. Outro aspecto que merece destaque é o fato de que as estimativas associadas à camada-3 e camada-4, obtidas a partir do emprego dos métodos da amplitude e logarítmico, são novamente bastante semelhantes.

De maneira geral, em consequência do elevado total pluviométrico, as mais elevadas magnitudes desta grandeza estão associadas ao ano de 2011. Com relação a este ano, ao contrário do que se esperava levando em consideração o total pluviométrico, as máximas não estiveram associadas ao mês de Maio (a precipitação deste superou significativamente a registrada nos demais meses). De acordo com Prevedello (1996), a difusividade térmica do solo eleva-se com o aumento do teor de umidade do solo, até alcançar certo valor máximo. A partir desse momento, a condutividade térmica do solo não mais se eleva com o aumento da umidade do solo e, por conseguinte, a difusividade térmica decresce.

Em uma primeira análise das referidas tabelas, com exceção do caso particular associado à camada-4, não se pode determinar com exatidão o método que menos se adéqua a estimativa desta grandeza física. Uma maneira eficaz de determinar a precisão dos métodos empregados consiste em, fazendo uso das estimativas da difusividade térmica, simular as temperaturas para diferentes horários e profundidade do solo a partir da Equação 1. Temperaturas próximas daquelas mensuradas indica que o método se adéqua a estimativa desta grandeza física. As ferramentas estatísticas utilizadas, como mencionado anteriormente na metodologia, têm como objetivo indicar o quanto as temperaturas mensuradas se aproximam daquelas simuladas. As magnitudes destas, para cada profundidade e método empregado, são expostas na Tabela 4.

Verifica-se, a partir da análise da Tabela 4, que as temperaturas simuladas estão fortemente correlacionadas aquelas mensuradas. Para o ano de 2010, fazendo uso das estimativas oriundas do método do arco tangente para a profundidade de $50 \mathrm{~cm}$, chegou-se a obter uma correlação em torno dos $99 \%$. De acordo com o índice "d", similarmente ao verificado por Otunla e Oladiran (2013), há uma concordância quase que perfeita entre as temperaturas simuladas e aquelas mensuradas. A concordância entre ambas, com exceção daquela associada à profundidade de $15 \mathrm{~cm}$, encontra-se superior a 97\%. Portanto, ambos os índices estatísticos indicam que as temperaturas simuladas são significativamente semelhantes 
aquelas mensuradas. Estes resultados, visto que a única variável necessária para simular as temperaturas são as magnitudes da difusividade térmica, demonstram que os métodos utilizados forneceram excelentes estimativas. O desempenho da Equação 1, de acordo com o índice de confiança, é ótimo para a simulação da temperatura do solo. Portanto, mesmo sendo uma das maneiras mais simples de representar o regime térmico diário do solo para diferentes profundidades, mostrou-se bastante eficaz o emprego desta equação.

Tabela 4. Resultados obtidos a partir do emprego das seguintes ferramentas estatísticas: coeficiente de correlação "r", índice de concordância "d" e índice de confiança "c".

\begin{tabular}{|c|c|c|c|c|c|c|}
\hline \multirow{2}{*}{ Ano } & \multirow{2}{*}{ Método } & \multirow{2}{*}{$\begin{array}{c}\text { Ferramenta } \\
\text { Estatística }\end{array}$} & \multicolumn{4}{|c|}{ Profundidade } \\
\hline & & & $5 \mathrm{~cm}$ & $15 \mathrm{~cm}$ & $30 \mathrm{~cm}$ & $50 \mathrm{~cm}$ \\
\hline \multirow[t]{9}{*}{2010} & Amplitude & $\mathrm{r}$ & 0,9553 & 0,9762 & 0,9749 & 0,9822 \\
\hline & & d & 0,9765 & 0,9564 & 0,9833 & 0,9872 \\
\hline & & $\mathrm{c}$ & Ótimo & Ótimo & Ótimo & Ótimo \\
\hline & Arco Tangente & $\mathrm{r}$ & 0,9567 & 0,9789 & 0,9726 & 0,9908 \\
\hline & & d & 0,9779 & 0,9575 & 0,9819 & 0,9925 \\
\hline & & $\mathrm{c}$ & Ótimo & Ótimo & Ótimo & Ótimo \\
\hline & Logarítmico & $\mathrm{r}$ & 0,9555 & 0,9768 & 0,9817 & 0,9785 \\
\hline & & $\mathrm{d}$ & 0,9767 & 0,9567 & 0,9877 & 0,9850 \\
\hline & & $\mathrm{c}$ & Ótimo & Ótimo & Ótimo & Ótimo \\
\hline \multirow[t]{9}{*}{2011} & Amplitude & $\mathrm{r}$ & 0,9526 & 0,9761 & 0,9577 & 0,9648 \\
\hline & & $\mathrm{d}$ & 0,9754 & 0,9309 & 0,9734 & 0,9764 \\
\hline & & $\mathrm{c}$ & Ótimo & Ótimo & Ótimo & Ótimo \\
\hline & Arco Tangente & $\mathrm{r}$ & 0,9513 & 0,9709 & 0,9611 & 0,9863 \\
\hline & & d & 0,9750 & 0,9323 & 0,9754 & 0,9884 \\
\hline & & $\mathrm{c}$ & Ótimo & Ótimo & Ótimo & Ótimo \\
\hline & Logarítmico & $\mathrm{r}$ & 0,9526 & 0,9765 & 0,9663 & 0,9606 \\
\hline & & d & 0,9755 & 0,9315 & 0,9788 & 0,9742 \\
\hline & & $\mathrm{c}$ & Ótimo & Ótimo & Ótimo & Ótimo \\
\hline
\end{tabular}

\section{CONCLUSÕES}

O solo apresenta um regime térmico diário e anual bem definido.

$\mathrm{O}$ método do arco tangente, para o caso específico da camada compreendida entre $5 \mathrm{e}$ $50 \mathrm{~cm}$ de profundidade, não é adequado para a estimativa da difusividade térmica do solo.

Os demais métodos mostram-se amplamente eficazes para estimativa da difusividade térmica do solo.

A equação destinada a simular as temperaturas do solo fornece resultados bastante satisfatórios.

\section{AGRADECIMENTOS}

Os autores agradecem ao Conselho Nacional de Desenvolvimento Científico e Tecnológico (CNPq) pela concessão de bolsa de estudo, bem como a Empresa Estadual de Pesquisa Agropecuária da Paraíba (EMEPA/PB) pelo fornecimento dos dados utilizados neste estudo. 


\section{REFERÊNCIAS}

ARAÚJO, T. S. Influência de elementos meteorológicos sobre o desenvolvimento da alface em diferentes ambientes. 2008. 93p. Dissertação (Mestrado em Meteorologia) Universidade Federal de Campina Grande, Campina Grande, 2008.

ARAÚJO, T. S.; FIDELES FILHO, J.; KUMAR, K. K.; RAO, T. V. R. Crescimento da alface-americana em função dos ambientes, épocas e graus-dias. Revista Brasileira de Ciências Agrárias, v. 5, p. 441-449, 2010. http://dx.doi.org/10.5039/agraria.v5i4a480

AZEVEDO, T. R.; GALVANI, E. Ajuste do ciclo médio mensal horário da temperatura do solo em função da temperatura do ar. Revista Brasileira de Agrometeorologia, v. 11, p. 123-130, 2003.

BELLAVER, V. Difusividade térmica do solo em área monodominante de cambará no norte do Pantanal Matogrossense. 2010. 70p. Dissertação (Mestrado em Física Ambiental) - Universidade Federal de Mato Grosso, Cuiabá, 2010.

CAMARGO, A. P.; SENTELHAS, P. C. Avaliação do desempenho de diferentes métodos de estimativa da evapotranspiração potencial no estado de São Paulo, Brasil. Revista Brasileira de Agrometeorologia, v. 5, p. 89-97, 1997.

CARNEIRO, R. G.; MOURA, M. A. L.; SILVA, V. P. R.; SILVA JUNIOR, R. S.; ANDRADE, A. M. D.; SANTOS, A. B. Variabilidade da temperatura do solo em função da liteira em fragmento remanescente de mata atlântica. Revista Brasileira de Engenharia Agrícola e Ambiental, v. 18, p. 99-108, 2014. http://dx.doi.org/10.1590/S1415-43662014000100013

DANELICHEN, V. H. M.; BIUDES, M. S. Avaliação da difusividade térmica de um solo no norte do Pantanal. Ciência e Natura, v. 33, p. 227-240, 2011.

DINIZ, J. M. T. Caracterização das propriedades térmicas do solo de Lagoa Seca-PB. 2014. 72p. Dissertação (Mestrado em Meteorologia) - Universidade Federal de Campina Grande, Campina Grande, 2014.

DINIZ, J. M. T.; ALBUQUERQUE, E. M.; RAFAEL, R. A. Estudo acerca da variabilidade térmica do solo. Scientia Plena, v. 10, p. 1-7, 2014.

DINIZ, J. M. T.; ALVINO, F. C. G.; SOUSA JÚNIOR, J. R.; WANDERLEY, J. A. C.; SOUSA, E. P.; MARACAJÁ, P. B. Características térmicas do solo de João Pessoa-PB. Agropecuária Científica no Semiárido, v. 9, p. 69-74, 2013 a.

DINIZ, J. M. T.; ARANHA, T. R. B. T.; SOUSA, E. P.; WANDERLEY, J. A. C.; SOUSA, E. P.; MARACAJÁ, P. B. Avaliação da difusividade térmica do solo de Campina GrandePB-Brasil. Agropecuária Científica no Semiárido, v. 9, p. 55-60, 2013 b.

DINIZ, J. M. T.; CARNEIRO, R. G.; ALVINO, F. C. G.; SOUSA, E. P.; SOUSA, E. P.; SOUSA JÚNIOR, J. R. Avaliação do comportamento térmico diário do solo de Campina Grande-PB. Agropecuária Científica no Semiárido, v. 9, p. 77-82, 2013c.

DINIZ, J. M. T.; RAFAEL, R. A.; FIDELES FILHO, J.; SOUSA JÚNIOR, J. R.; FERNANDES, A. A. Características térmicas do solo observadas em cidades distintas do estado da Paraíba. Revista Verde de Agroecologia e Desenvolvimento Sustentável, v. 8, p. 117-126, $2013 \mathrm{~d}$. 
DINIZ, J. M. T.; SOUSA, E. P.; WANDERLEY, J. A. C.; FIDELES FILHO, J.; MARACAJÁ, P. B. Variabilidade diária da temperatura do solo: um estudo de caso. Revista Verde de Agroecologia e Desenvolvimento Sustentável, v. 8, p. 01-06, 2013 e.

FIDELES FILHO, J. Estrutura térmica de solos do Nordeste do Brasil. 1988. 85p. Dissertação (Mestrado em Meteorologia) - Universidade Federal da Paraíba, Campina Grande, 1988.

GAO, Z.; WANG, L.; HORTON, R. Comparison of six algorithms to determine the soil thermal diffusivity at a site in the Loess Plateau of China. Hydrology and Earth System Sciences Discussions, v. 6, p. 2247-2274, 2009.

http://dx.doi.org/10.5194/hessd-6-2247-2009

GASPARIM, E.; RICIERI, R. P.; SILVA, S. L.; DALlACORT, R.; GNOATTO, E. Temperatura no perfil do solo utilizando duas densidades de cobertura e solo nu. Acta Scientiarum, v. 27, p. 107-115, 2005.

http://dx.doi.org/10.4025/actasciagron.v27i1.2127

HILLEL, D. Introduction to environmental soil physics. Amsterdam: Elsevier Academic Press, 2004. 494p.

LAL, R.; SHUKLA, M. K. Principles of soil physics. New York: Marcel Dekker Inc., 2004. $716 \mathrm{p}$.

LARSON, R.; FARBER, B. Estatística aplicada. São Paulo: Prentice Hall, 2004. 476p.

OLIVEIRA, S. S.; FIDELES FILHO, J.; OLIVEIRA, S. V.; ARAÚJO, T. S. Difusividade térmica do solo de Campina Grande para dois períodos do ano. Revista de Geografia, v. 27, p. 179-189, 2010.

OTUNLA, T. A.; OLADIRAN, E. O. Evaluation of soil thermal diffusivity algorithms at two equatorial sites in West Africa. Annals of Geophysics, v. 56, p. 1-12, 2013. http://dx.doi.org/10.4401/ag-6170

PEDRON, F. A. Mineralogia, morfologia e classificação de saprolitos e neossolos derivados de rochas vulcânicas no Rio Grande do Sul. 2007. 160p. Tese (Doutorado em Ciência do Solo) - Universidade Federal de Santa Maria, Santa Maria, 2007.

PREVEDELLO, C. L. Física do solo com problemas resolvidos. Curitiba: SaleswardDiscovery, 1996. 446p.

RAO, T. V. R.; SILVA, B. B.; MOREIRA, A. A. Características térmicas do solo em Salvador, BA. Revista Brasileira de Engenharia Agrícola e Ambiental, v. 9, p. 554559, 2005. http://dx.doi.org/10.1590/S1415-43662005000400018

REICHARDT, K.; TIMM, L. C. Solo, planta e atmosfera: conceitos, processos e aplicações. Barueri: Manole, 2004. 478p.

SILANS, A. P.; SILVA, F. M.; BARBOSA, F. A. R. Determinação in loco da difusividade térmica num solo da região de caatinga (PB). Revista Brasileira de Ciência do Solo, v. 30, p. 41-48, 2006. http://dx.doi.org/10.1590/S0100-06832006000100005

TITO, G. A.; CHAVES, L. H. G.; CARVAlHO, H. O.; AZEVEDO, N. C. Aplicação de bentonita em um regossolo eutrófico. II. Efeitos sobre as propriedades químicas do solo. Revista Brasileira de Engenharia Agrícola e Ambiental, v. 1, p. 25-27, 1997. 
WILLMOTT, C. J.; ACKLESON, S. G.; DAVIS, R. E.; FEDDEMA, J. J.; KLINK, K. M.; LEGATES, D. R. et al. Statistics for the evaluation and comparison of models. Journal of Geophysical Research, v. 90, p. 8995-9005, 1985.

http://dx.doi.org/10.1029/JC090iC05p08995

ZONTA, J. H.; MARTINEZ, M. A.; SILVA, D. D.; PRUSKI, F. F.; SANTOS, M. R. Modelagem da infiltração em solos com encrostamento superficial. Parte II: Condutividade hidráulica variando no tempo. Revista Brasileira de Engenharia Agrícola e Ambiental, v. 16, p. 471-479, 2012. http://dx.doi.org/10.1590/S141543662012000500002 